\title{
Genetic Analysis for Forage Yield and Morphological Traits of Seed in Oat (Avena sativa L.)
}

\author{
Atar Singh ${ }^{1}$, Mayank Chaudhary ${ }^{2 *}$, Nirdesh K. Chaudhary ${ }^{2}$ and Chiranjeev ${ }^{2}$ \\ ${ }^{1}$ Department of GPB, Chandra Shekhar Azad University of Agriculture \& Technology, \\ Kanpur, U.P., India \\ ${ }^{2}$ Department of GPB, Sardar Vallabhbhai Patel University of Agriculture \& Technology, \\ Meerut, U.P., India \\ *Corresponding author
}

\section{A B S T R A C T}

\section{Keywords}

cereal annual crop, silicon, Manganese, Zinc, Calcium, Phosphorus

\section{Article Info}

Accepted: 04 September 2019 Available Online: 10 October 2019
Present investigation was carried out at the Students Instructional Farm of Chandra Shekhar Azad University of Agriculture and Technology, Kanpur during Rabi-201718. Analysis of variance revealed significant variation exists among the stains for all characters studied except stem girth, leaf width, nodes per plant, leaves per plant and seedlings dry weight. Heritability estimates varied from 6.65 percent for leaves per plant to 98.33 percent for days to maturity. Seed vigour index showed high GCV. Moderately high variability for GCV coupled with high estimates of heritability were observed for seedlings dry weight, green fresh weight of total tillers and green leaves weight per plant. Green fresh weight of total tillers exhibited comparatively higher estimates of genotypic coefficient variance, heritability and genetic advance as percent of mean which is indicative of predominance of additive gene action in expression of this trait therefore, for this character selection appears to be effective. The genetic advancement as percent mean was found to be highest for green fresh weight of total tillers (61.00) and moderately observed for plant height. This study will provide opportunity to identify best genotypes to be used in breeding. Besides it helps in understanding the diversity available in the genotypes selected and helps in selection and improvement of desirable traits to be used or transferred during crossing programme.

\section{Introduction}

Oats (Avena sativa L.) is the most important cereal annual crop of belongs to family gramineae grown in rabi season in several states of country including north western, central and extending up to the states of eastern India. Oat has sixth ranks in cereal production globally following wheat, maize, rice, barley and sorghum. It is considered to be one of the best dual purpose cereal crop that fit well into the society of human and population cattle as well. In respect to consumption by human and cattle feeding 
purposes, high nutrients contents of protein, carbohydrates, lipids, silicon, manganese, zinc, calcium, phosphorus and vitamin A, B1, $\mathrm{B} 2, \mathrm{E}$ and lower fiber contents are required. Oats taxonomic patters are similar to that of wheat and consists of polyploidy series with seven $(n=7)$ chromosome numbers i.e. diploid $(2 \mathrm{n}=2 \mathrm{x}=14)$, tetraploid $(2 \mathrm{n}=4 \mathrm{x}=28)$ and hexaploid $(2 n=6 x=42)$ The common oat (Avena sativa L.) is grown in India as dual purpose crop with the total area of about 500,000 hectares is covered under oat cultivation in the country. The crop occupies maximum area in Uttar Pradesh (34\%), followed by Punjab (20\%), Bihar (16\%), Haryana (9\%) and Madhya Pradesh (6\%). Rest of the area is shared by other states i.e. Gujarat, Maharashtra, Odessa, Uttrakhand etc. (Annonymous,2015).Presently India faces a net deficit of $63 \%$ green fodder, $24 \%$ dry fodder residues and $64 \%$ feeds due to increasing population of cattle's and as point of view of better production by animals. This crop gives heavy yield and the average yield of seed and green fodder ranges from 1.5 to 2.5 and 45 to 55 tons per hectare respectively. Yield of any crop is a complex and quantitatively inherited character, contributed by the various characters and influenced by environmental variation and thus considering the importance of effecting improvement in the seed and fodder yield characters and its contributing traits primarily depends on nature and magnitude of the heritable traits.

\section{Materials and Methods}

An experiment was conducted at Students Instructional Farm of Chandra Shekhar Azad University of Agriculture and Technology, Kanpur during Rabi-2017-18. The research materials comprised twenty five number of genotypes of diverse nature namely, CSOFSC12-2, CSOFSC-11-5, Kent, CSOFSC-11-4, CSOFSC-11-1, CSOFSC-12-1, CSOFSC-121, UPO-212, ANDO-1, JHO-03-91, CSAOSC-
12-1, ANDO-2, OS-403, OS-344, OS-1, SKO105, NDO-25, JHO-2007-2, CSAOSC-14-6, SKO-105, JHO-2007-2, JHO-03-93, NDO612, OS-6, JHO-851 and JHO-99-2 were evaluated in RBD with three replications with row to row spacing of $30 \mathrm{~cm}$ under late sown condition and In order to test the validity of performance of each varieties statistical analysis was done in accordance to Randomized Completely Block Design (RCBD) for all the field and laboratory observations. Observations were recorded on five randomly selected plants in each replication for green fresh weight at 50 days (g),days to $50 \%$ flowering, green plant weight $(\mathrm{g})$, green leaves weight per plant (g) stem girth $(\mathrm{cm})$, total numbers of tillers per plant, leaf length $(\mathrm{cm})$, leaf width $(\mathrm{cm})$, number of nodes per plant, number of leaves per plant, plant height $(\mathrm{cm}$.$) , days to maturity, panicle$ length $(\mathrm{cm})$, biological yield per plant $(\mathrm{g})$ seeds per plant,100 seed weight $(\mathrm{g})$, dry weight per plant (g), Harvest index (\%), seed germination in percent, seedling length $(\mathrm{cm})$ seedling dry weight $(\mathrm{g})$, seed vigour index and seed yield per plant $(\mathrm{g})$.The data for quantitative characters were subjected to analysis of variance (ANOVA) for randomized completely block design statistically analyzed The differences between treatments means were compared using ' $\mathrm{F}$ ' value at $1 \%$ and $5 \%$ probability levels.

Estimation of Variability: Different parameters such as mean, range coefficient of variation etc. were used to estimate to the diversity present among the genotypes for different quantitative traits and genotypic variances and coefficients of variation using formula as suggested by Burton and de Vane (1953) as:

Genotypic variance $\left(\sigma^{2} \mathrm{~g}\right)$

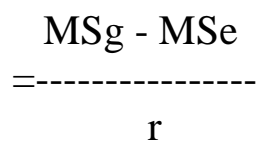


Where, $r$ is numbers of replications, MSg is mean square due to genotypes, MSe is mean square of error (Environmental variance), Environmental variance $\left(\sigma^{2} \mathrm{e}\right)$ is error mean square, Phenotypic variance $\left(\sigma^{2} p\right)$ is $\sigma^{2} g+$ MSe where, $\sigma^{2} \mathrm{~g}$ is genotypic variance and phenotypic coefficient variation (PCV) estimated as the following formula:

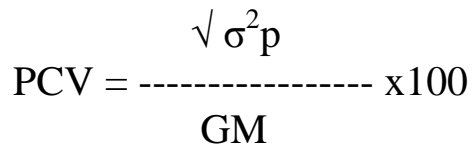

Where, GM is an overall mean of character and $\sigma^{2} \mathrm{p}$ is $\sigma^{2} \mathrm{~g}+\mathrm{MSe}$

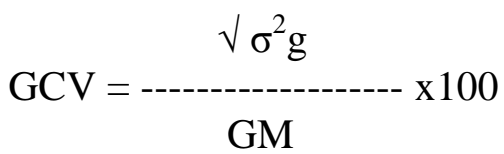

Where, GM is an overall mean of character and $\sigma^{2} \mathrm{~g}$ is $\mathrm{MSg}+\mathrm{MSe} /$ Replications

Estimation of Heritability: Heritability is the ratio of the genotypic variance to the total variance i.e. phenotypic variance (genotypic and environmental), and it denotes the proportion of phenotypic variance that is due to genotypes i.e., heritable and calculated as per the formula given by (Hasan et al., 1956).

$\mathrm{H}($ board sense $)=\frac{\sigma^{2} \mathrm{~g}}{\sigma_{\mathrm{p}}^{2}}$

Where

$\sigma^{2} \mathrm{~g}=$ Genotypic variance

$\sigma_{\mathrm{p}}^{2}=$ phonotypic variation (Variance genotypic + variance environmental)

Estimation of Genetic Advance: It is the improvements in the mean genotypic value of the selected families over the base population.

Genetic advance was calculated in percents of mean according to Johnson et al.,(1955a). as given here under:
GA $(\%$ of mean $)=\frac{\sigma^{2} \mathrm{~g} \times(\mathrm{K})}{\left(\sigma^{2} \rho\right) \times \text { mean }}$

Where

$\mathrm{K}$, Selection differential at $5 \%$ selection intensity $(\mathrm{K}=2.06)$

$\sigma^{2} \mathrm{~g}=$ Genotypic variance

$\sigma^{2} \rho=$ Phenotypic stand and deviation of the character

\section{Results and Discussion}

\section{Variability analysis}

The analysis of variance revealed a significant variation (Table 1) among the genotypes for all traits green fresh weight $\left(392.85^{* *}\right)$, days to flowering $(63.97 * *)$, number of total tillers per plant $(26.53 * *)$, green plant weight $(77.39 * *)$, green leaves weight per plant $\left(5.90^{* *}\right)$, leaf length $\left(39.59^{* *}\right)$, days to maturity $\left(16.63^{* *}\right)$, plant height $(147.03 * *)$, panicle length $\left(30.13^{* *}\right)$, biological yield per plant $\left(2.77^{* *}\right)$, seeds per plant $(70.69 * *), 100$ seed weight $\left(1.20^{*}\right)$, dry weight per plant $\left(2.47^{* *}\right)$, harvest index $\left(33.05^{* *}\right)$ seed germination in percent $\left(69.25^{* *}\right)$, seedling length $(36.62 * *)$, seed vigour index (1.288) and seed yield per plant $(0.51 *)$, except stem girth (0.22), leaf width (0.02), number of nodes per plant (0.02), number of leaves per plant (0.03) and seedlings dry weight per plant (0.2) under investigation, there by indicating the existence of a considerable magnitude to genetic variability among the genotypes. (Khan et al.,(2002), Wani et el. (2013), Krishana et al., (2013), Bajpai et al., (2014) and Singh et al., (2018). The mean values of traits, range, phenotypic coefficient of variation (PCV), genotypic coefficient of variation (GCV), heritability, and genetic advance as percent of mean at $5 \%$ are mentioned in Table 2 and 3 respectively. Estimation of range of mean for all the characters studied where, a wide range for 
green fresh weight of total tillers was observed 82.60 to 201.80. with lowest in variety CSOFSC-11-1 and highest in SKO -105 with an overall mean value (151.85), coefficient of variation (9.71), standard error (12.04) and critical distance (24.21). Days to flowering had a range of 83.93 to 101.80 with lowest in variety JHO-03-91 and highest in NDO- 25 with an overall mean value(93.85),coefficient of variation (0.53), standard error (0.29) and critical difference (0.82). Total number of tillers per plant having a range of 8.53 to 20.13 with lowest in genotype CSOFSC-11-1 and highest in SKO105 with an overall mean value (14.40), coefficient of variation (7.82), standard error (0.65) and critical distance (1.85). Green weight of per plant was showed a range of 16.33 to 36.00 with lowest in variety OS344 and highest in CSAOSC-14-6 with an overall mean value (28.08), coefficient of variation (12.74), standard error (2.07) and critical distance (5.87). The green leaves weight per plant was exhibited a range of 2.27 to 8.52 , with minimum value of mean in variety OS-344 and maximum in OS-6 as compared to mean value (5.99), coefficient of variation (16.64), standard error (0.57) and critical difference (1.63). The character stem girth was showed a range of 1.70 to 2.90 with lowest in variety OS-344 and highest in CSAOSC-14-6 with an overall mean value (2.24), coefficient of variation (7.00), standard error (0.09) and critical distance (0.26). Leaf length exhibited a range of 35.87 to 50.07; with lower value of mean in genotype CSOFSC12-2 and higher in ANDO-2 with an overall mean value (44.43), coefficient of variation (3.17), standard error (0.81) and critical difference (2.31). The character leaf width displayed a range of 1.48 to 1.81 with lowest mean value in an accession CSAOSC12-1 and highest in Kent with an overall mean value (1.66), coefficient of variation (2.37), standard error (0.02) and critical distance (0.06). Number of nodes per plant displayed a range of 5.20 to 5.47 with lowest in variety JHO03-93 and highest in CSOFSC11-4 with an overall mean value (5.35), coefficient of variation (3.45), standard error $(0.11)$ and critical distance $(0.80)$. The range of 5.20 to 5.47 with lowest in variety JHO-03-93 and highest in CSOFSC11-1 with an overall mean value (5.34), coefficient of variation (3.70), standard error (0.11) and critical distance (0.33) were recorded for leaves per plant. Days to maturity had a range of 123.67 to 131.07 with lowest in variety CSOFSC12-2 and highest in ANDO-1with an overall mean value (127.51), coefficient of variation (0.24), standard error (0.18) and critical distance (0.50). The plant height expressed a mean values of 154.67 to 177.93 with lowest in variety SKO105 and highest in CSOFSC11-5 with an overall mean value (167.74), coefficient of variation (1.78), standard error (1.72) and critical distance (4.89). Panicle length had a range of 17.13 to 29.05 with lowest in variety JHO851 and highest in Kent with an overall mean value (25.68), coefficient of variation (2.79), standard error (0.01) and critical distance (1.17). The range of 12.53 to 16.33 , with lowest in variety Kent and highest in UPO212 with an overall mean value (14.22), coefficient of variation (1.34), standard error (0.11) and critical distance (0.31) were observed for this character. For seeds per plant were recorded estimations of variations viz., range from 88.27 to 106.27 with lowest in genotype OS6 highest in UPO-212 with an overall mean value (96.02), coefficient of variation (1.10), standard error (0.61) and critical distance (1.74). The character 100 - seeds weight showed a range from 3.21to 5.19 with lowest in variety OS-1 highest in NDO-612 with an overall mean value (4.25), coefficient of variation (6.77), standard error (0.17) and critical distance (0.47). Dry weight per plant was showed a range of 8.68 to 11.72 with lowest in variety CSAOSC-14-6 and highest in CSAOSC12-1 with an overall mean value (10.09), coefficient of variation (2.37), 
standard error (0.14) and critical distance (0.39). Harvest index displayed a range of 25.75 to $37.22(\%)$ with lowest in variety JHO03-93 and highest for JHO-2007-2 with an overall mean value (29.70), coefficient of variation (2.46), standard error (0.42) and critical distance (1.20). The variability estimations namely; range of 65.40 to 90.77 with lowest in variety CSOFSC12-2 and highest in SKO-105 with an overall mean value (84.47), coefficient of variation (8.15), standard error (3.98) and critical distance (11.31) were observed for seed germination in percent. The character seedling length per plant showed a wide range of 18.43 to 28.47 with lowest in genotype CSOFSC11-1 and highest in SKO-105 with an overall mean value (23.05), coefficient of variation (5.32), standard error (0.71) and critical distance (2.01). The single seedling dry weight displayed a range of 0.02 to 0.04 , with lowest in JHO-03-93 and highest in CSOFSC-12-1 with an overall mean value (0.03), coefficient of variation (13.51), standard error (0.0) and critical distance (0.0). For seed vigour index a range of 1.49 to 4.02 with lowest in variety JHO99-2 and highest in JHO03-91 with an overall mean value (2.37), coefficient of variation (13.87), standard error (0.27) and critical distance $(0.54)$ were observed. Seed yield per plant exhibited a range of 3.68 to 5.28 with lowest in variety $\mathrm{JHO} 03-93$ and highest in JHO-2007-2 with an overall mean value (4.20), coefficient of variation (2.16), standard error (0.05) and critical distance (0.15). A crossing between genotypes exhibiting significant variance and higher value of mean for the desired characters will help in development of variety with increase in seed and green, dry fodder yield. Khan et al., (2002), Bibi et al., (2012), Krishana et al.,(2013), Dubey et al., (2014), Bajpai et al., (2014), Kumar et al., (2017) and Singh et al., (2018).

Table.1 Analysis of variance for different traits in genotypes of oat (Avena sativa L.)

\begin{tabular}{|c|c|c|c|c|c|c|c|c|c|c|c|c|c|}
\hline S.V. & d. f. & $\begin{array}{c}\text { GFWTT } \\
\text { (g) }\end{array}$ & DF & TTPP & $\begin{array}{c}\text { GWPP } \\
\text { (g) }\end{array}$ & $\begin{array}{c}\text { GLWPP } \\
\text { (g) }\end{array}$ & SG (cm) & $\mathbf{L L}(\mathbf{c m})$ & $\mathbf{L W}(\mathbf{c m})$ & NPP & LPP & DM & $\mathrm{PH}(\mathrm{cm})$ \\
\hline Treatment & 24 & $392.85 * *$ & $\begin{array}{c}63.97 * \\
*\end{array}$ & $26.53^{* *}$ & $77.39 * *$ & $5.90 * *$ & 0.22 & $39.59 * *$ & 0.02 & 0.02 & 0.03 & $16.63 * *$ & $147.03 * *$ \\
\hline Replication & 2 & 198.41 & 4.98 & 0.19 & 22.23 & 0.92 & 0.26 & 120.07 & 0.02 & 0.13 & 0.18 & 8.65 & 1169.73 \\
\hline Error & 48 & 217.51 & 0.25 & 1.27 & 12.81 & 0.99 & 0.02 & 1.98 & 0.00 & 0.03 & 0.04 & 0.09 & 8.89 \\
\hline
\end{tabular}

\begin{tabular}{|c|c|c|c|c|c|c|c|c|c|c|c|c|}
\hline S.V. & d. f. & PL(cm) & BYPP(g) & SPP & HSW & DWPP(g) & HI (\%) SG (\%) & SLL(cm) & SLDW(g) & SVI & SYPP(g) \\
\hline Treatment & 24 & $30.13^{* *}$ & $2.77 * *$ & $70.69 * *$ & $1.20^{*}$ & $2.47 * *$ & $33.05^{* *}$ & $69.25 * *$ & $36.62^{* *}$ & 0.2 & $1.28^{*}$ & $0.51^{* *}$ \\
\hline Replication & 2 & 17.83 & 7.43 & 21.87 & 0.07 & 3.86 & 14.89 & 44.70 & 1.11 & 0.3 & 0.19 & 0.05 \\
\hline Error & 48 & 0.51 & 0.04 & 1.12 & 0.08 & 0.06 & 0.53 & 47.45 & 1.50 & 0.1 & 0.11 & 0.01 \\
\hline
\end{tabular}

$*, * *$ significant at $5 \%$ and $! \%$ levels, respectively. 
Table.2 Mean, coefficient of variation, standard error and critical distance for various, seed and fodder yield and related traits in oat (Avena sativa L.)

\begin{tabular}{|c|c|c|c|c|c|c|c|c|c|c|c|c|}
\hline $\begin{array}{c}\text { Traits/pa } \\
\text { rameters }\end{array}$ & $\begin{array}{c}\text { GFWT } \\
\text { T(g) }\end{array}$ & DF & TTPP & $\begin{array}{c}\text { GWP } \\
\mathbf{P ( g )}\end{array}$ & $\begin{array}{c}\text { GLWP } \\
\mathbf{P}(\mathbf{g})\end{array}$ & $\begin{array}{c}\text { SG } \\
(\mathbf{c m})\end{array}$ & $\begin{array}{c}\text { LL } \\
(\mathbf{c m})\end{array}$ & $\begin{array}{c}\text { LW } \\
(\mathbf{c m})\end{array}$ & NPP & LPP & DM & $\begin{array}{c}\text { PH } \\
(\mathbf{c m})\end{array}$ \\
\hline Mean & 151.85 & 93.85 & 14.40 & 28.08 & 5.99 & 2.24 & 44.43 & 1.66 & 5.35 & 5.34 & 127.51 & 167.74 \\
\hline C.V. & 9.71 & 0.53 & 7.82 & 12.74 & 16.64 & 7.00 & 3.17 & 2.37 & 3.45 & 3.70 & 0.24 & 1.78 \\
\hline F.Ratio & 15.60 & 257.74 & 20.90 & 6.04 & 5.94 & 8.85 & 20.00 & 15.93 & 0.56 & 0.81 & 177.71 & 16.54 \\
\hline S.E.(m) & 8.51 & 0.29 & 0.65 & 2.07 & 0.57 & 0.09 & 0.81 & 0.02 & 0.11 & 0.11 & 0.18 & 1.72 \\
\hline C.D.5\% & 24.21 & 0.82 & 1.85 & 5.87 & 1.63 & 0.26 & 2.31 & 0.06 & 0.30 & 0.33 & 0.50 & 4.89 \\
\hline
\end{tabular}

\begin{tabular}{|c|c|c|c|c|c|c|c|c|c|c|c|}
\hline $\begin{array}{c}\text { Traits/pa } \\
\text { rameters }\end{array}$ & PL(cm) & $\begin{array}{l}\text { BYP } \\
\mathbf{P}(\mathbf{g})\end{array}$ & SPP & HSW & $\begin{array}{c}\text { DWPP } \\
(\mathbf{g})\end{array}$ & $\begin{array}{c}\text { HI } \\
(\boldsymbol{\%})\end{array}$ & $\begin{array}{c}\text { SG } \\
(\boldsymbol{\%})\end{array}$ & SLL(cm) & $\begin{array}{c}\text { SSLD } \\
\text { W(g) }\end{array}$ & SVI & SYPP(g) \\
\hline Mean & 25.68 & 14.22 & 96.02 & 4.25 & $10 . .09$ & 29.70 & 84.47 & 23.05 & 0.03 & 2.37 & 4.20 \\
\hline C.V. & 2.79 & 1.34 & 1.10 & 6.77 & 2.37 & 2.46 & 8.15 & 5.32 & 13.51 & $\begin{array}{c}13.8 \\
7\end{array}$ \\
\hline F.Ratio & 58.83 & 75.90 & 62.88 & 14.44 & 43.34 & 61.83 & 1.46 & 24.37 & 20.00 & 11.8 & 61.55 \\
\hline S.E.(m) & 0.41 & 0.11 & 0.61 & 0.17 & 0.14 & 0.42 & 3.98 & 0.71 & 0.00 & 0.19 & 0.05 \\
\hline C.D.5\% & 1.17 & 0.31 & 1.74 & 0.47 & 0.39 & 1.20 & 11.31 & 2.01 & 0.00 & 0.54 & 0.15 \\
\hline
\end{tabular}




\begin{tabular}{|c|c|c|c|c|c|c|c|c|c|}
\hline \multirow[t]{2}{*}{ Parameters/ Traits } & \multicolumn{2}{|c|}{ Range } & \multirow[t]{2}{*}{$\sigma^{2} \mathrm{e}$} & \multirow[t]{2}{*}{$\sigma^{2} g$} & \multirow[t]{2}{*}{ GCV } & \multirow[t]{2}{*}{$\sigma^{2} p$} & \multirow[t]{2}{*}{ PCV } & \multirow[t]{2}{*}{$\mathbf{H}^{2}(\mathrm{bs})$} & \multirow{2}{*}{$\begin{array}{c}\text { Gen.Adv. as\% } \\
\text { mean } 5 \%\end{array}$} \\
\hline & Min & Max & & & & & & & \\
\hline GFWTT(g) & 82.60 & 201.80 & 217.51 & 1058.44 & 21.43 & 1275.96 & 23.52 & 82.95 & 61.00 \\
\hline DF & 83.93 & 101.80 & 0.25 & 21.24 & 4.91 & 21.49 & 4.94 & 98.84 & 9.40 \\
\hline TTPP & 8.53 & 20.13 & 1.27 & 8.42 & 20.15 & 9.69 & 21.61 & 86.90 & 5.6 \\
\hline GPW(g) & 16.33 & 36.00 & 12.81 & 21.53 & 16.52 & 34.33 & 20.87 & 62.70 & 7.6 \\
\hline $\mathbf{L L}(\mathbf{c m})$ & 35.87 & 50.07 & 1.98 & 12.54 & 7.97 & 14.52 & 8.58 & 86.36 & 6.8 \\
\hline $\mathbf{L W}(\mathbf{c m})$ & 1.48 & 1.81 & 0.01 & 0.01 & 5.29 & 0.01 & 5.80 & 83.27 & 0.2 \\
\hline NPP & 5.2 & 5.47 & 0.03 & 0.01 & 0.01 & 0.03 & 3.18 & 17.41 & 0.1 \\
\hline LPP & 5.2 & 5.6 & 0.04 & 0.01 & 0.01 & 0.04 & 3.59 & 6.65 & 0.01 \\
\hline SPP & 88.27 & 106.27 & 1.12 & 23.19 & 5.01 & 24.31 & 5.14 & 95.38 & 9.7 \\
\hline HSW & 3.21 & 5.19 & 0.08 & 0.37 & 14.34 & 0.45 & 15.86 & 8.75 & 1.1 \\
\hline $\operatorname{DWPP}(\mathrm{g})$ & 8.68 & 11.72 & 0.06 & 0.81 & 8.89 & 0.86 & 9.20 & 93.38 & 1.8 \\
\hline HI(\%) & 25.75 & 37.22 & 0.53 & 10.84 & 11.09 & 11.37 & 11.37 & 95.30 & 6.6 \\
\hline SG (\%) & 65.40 & 90.77 & 47.45 & 7.27 & 3.19 & 54.71 & 8.76 & 13.29 & 2.0 \\
\hline SLL(cm) & 18.43 & 28.47 & 1.5 & 11.71 & 14.84 & 13.21 & 15.77 & 88.62 & 6.6 \\
\hline $\operatorname{SLDW}(\mathbf{g})$ & 0.02 & 0.04 & 0.00 & 0.00 & 24.29 & 0.00 & 27.79 & 76.37 & 0.0 \\
\hline SVI & 1.49 & 4.02 & 0.11 & 0.39 & 26.38 & 0.50 & 29.81 & 78.36 & 1.1 \\
\hline SYPP(g) & 3.68 & 5.28 & 0.01 & 0.17 & 9.72 & 0.18 & 9.96 & 95.28 & 0.8 \\
\hline
\end{tabular}


Table.3 estimates of different genetic parameters for various traits in oat (Avena sativa L.)

\begin{tabular}{|c|c|c|c|c|}
\hline $\begin{array}{c}\text { 1-GFWTT }(g)=\text { Green } \\
\text { fresh weight of Total } \\
\text { Tillers }(g)\end{array}$ & $\begin{array}{c}\text { 6-SG } \\
(\mathrm{cm})=\text { Stem } \\
\text { Girth }(\mathrm{cm})\end{array}$ & $\begin{array}{l}\text { 11-DM = Days to } \\
\text { maturity }\end{array}$ & $\begin{array}{c}\text { 16-HSW= 100- } \\
\text { Seeds weight }(g)\end{array}$ & $\begin{array}{c}\text { 21-SLDW }(g)= \\
\text { Seedling dry } \\
\text { weight }(g)\end{array}$ \\
\hline 2-DF = Days to flowering & $\begin{aligned} & 7-\mathrm{LL}(\mathrm{cm}) \\
= & \text { Leaf Length } \\
& (\mathrm{cm})\end{aligned}$ & $\begin{array}{l}\text { 12-PH }(\mathrm{cm})=\text { Plant } \\
\text { Height }(\mathrm{cm})\end{array}$ & $\begin{array}{l}17-\mathrm{DWPP}(\mathrm{g})=\text { Dry } \\
\text { weight per Plant }(\mathrm{g})\end{array}$ & $\begin{array}{l}22-S V I=\text { Seed } \\
\text { Vigour Index }\end{array}$ \\
\hline $\begin{array}{c}\text { 3-TTPP= Total Tillers } \\
\text { per Plant }\end{array}$ & $\begin{array}{c}8- \\
\text { LW }(\mathrm{cm})=\text { Leaf } \\
\text { width }(\mathrm{cm})\end{array}$ & $\begin{array}{l}\text { 13-PL }(\mathrm{cm}) \text { Panicle } \\
\text { Length }(\mathrm{cm})\end{array}$ & $\begin{array}{c}18-\mathrm{HI}(\%)=\text { Harvest } \\
\text { Index }(\%)\end{array}$ & \multirow[t]{3}{*}{$\begin{array}{l}23-\mathrm{SYPP}(\mathrm{g})=\text { Seed } \\
\text { yield per plant }(\mathrm{g})\end{array}$} \\
\hline $\begin{array}{c}\text { 4-GPW }(g)=\text { Green Plant } \\
\text { Weight }(g)\end{array}$ & $\begin{array}{l}\text { 9-NPP }=\text { Nodes } \\
\text { per PLANT }\end{array}$ & $\begin{array}{c}\text { 14-BYPP }(\mathrm{g})= \\
\text { Biological yield per } \\
\text { Plant }(\mathrm{g})\end{array}$ & $\begin{array}{l}19-\mathrm{SG}(\%)=\text { Seed } \\
\text { Germination }(\%)\end{array}$ & \\
\hline $\begin{array}{l}\text { 5-GLWPP(g) Green } \\
\text { leaves weight per Plant }(g)\end{array}$ & $\begin{array}{l}10-\mathrm{LPP}= \\
\text { Leaves per } \\
\text { Plant }\end{array}$ & $\begin{array}{c}15-\mathrm{SPP}=\text { Seeds per } \\
\text { Plant }\end{array}$ & $\begin{array}{l}\text { 20-SLL }(\mathrm{cm})= \\
\text { Seedling Length } \\
(\mathrm{cm})\end{array}$ & \\
\hline
\end{tabular}

$\sigma^{2} \mathrm{e}=$ Variance Environmental, $\sigma^{2} \mathrm{~g}=$ Variance Genotypic and $\sigma^{2} \mathrm{p}=$ Variance Phenotypic 
Table.4 Appendix -Mean per se performance for various character in genotypes of Oat ( Avena sativa l.)

\begin{tabular}{|c|c|c|c|c|c|c|c|c|c|c|c|c|}
\hline Variety & GFW & DF & TTPP & GPW & SG & GLW & $\mathbf{L L}$ & $\mathbf{L W}$ & NPP & LPP & DM & PH \\
\hline CSOFSC12-2 & 168.07 & 86.13 & 16.00 & 29.17 & 2.13 & 6.24 & 35.87 & 1.75 & 5.33 & 5.33 & 123.67 & 176.80 \\
\hline CSOFSC11-5 & 221.60 & 95.47 & 13.27 & 32.67 & 2.17 & 4.57 & 40.27 & 1.68 & 5.33 & 5.27 & 129.53 & 177.93 \\
\hline Kent & 181.40 & 94.20 & 16.27 & 28.50 & 2.45 & 6.57 & 43.67 & 1.81 & 5.33 & 5.27 & 128.13 & 172.87 \\
\hline CSOFSC11-4 & 118.13 & 96.60 & 17.33 & 19.67 & 2.47 & 6.29 & 36.07 & 1.72 & 5.47 & 5.20 & 128.00 & 177.47 \\
\hline CSOFSC11-1 & 82.60 & 95.67 & 8.53 & 21.00 & 2.53 & 3.25 & 38.27 & 1.64 & 5.33 & 5.47 & 129.80 & 177.60 \\
\hline CSOFSC12-1 & 199.00 & 95.13 & 19.53 & 28.00 & 2.73 & 4.48 & 44.60 & 1.60 & 5.33 & 5.33 & 128.07 & 167.60 \\
\hline UPO212 & 126.47 & 95.73 & 11.60 & 25.00 & 1.97 & 7.40 & 43.27 & 1.53 & 5.33 & 5.40 & 129.93 & 167.93 \\
\hline ANDO1 & 148.87 & 101.80 & 13.60 & 23.33 & 2.00 & 5.32 & 42.33 & 1.73 & 5.33 & 5.33 & 131.07 & 176.47 \\
\hline JHo03-91 & 159.67 & 83.93 & 15.13 & 32.33 & 2.10 & 7.92 & 46.07 & 1.61 & 5.27 & 5.33 & 124.87 & 166.73 \\
\hline CSAOSC12-1 & 99.40 & 93.80 & 9.87 & 25.00 & 2.00 & 5.63 & 45.53 & 1.48 & 5.47 & 5.33 & 129.07 & 176.40 \\
\hline ANDO2 & 114.07 & 95.00 & 10.20 & 28.00 & 2.40 & 5.41 & 50.07 & 1.60 & 5.47 & 5.27 & 129.60 & 163.27 \\
\hline OS403 & 136.47 & 94.20 & 12.93 & 32.67 & 2.23 & 5.57 & 49.87 & 1.69 & 5.33 & 5.53 & 131.00 & 164.67 \\
\hline OS344 & 150.20 & 93.73 & 12.73 & 16.33 & 2.03 & 2.27 & 45.80 & 1.53 & 5.40 & 5.53 & 130.13 & 167.80 \\
\hline OS1 & 148.33 & 100.13 & 13.80 & 24.33 & 2.13 & 6.98 & 44.40 & 1.73 & 5.40 & 5.40 & 128.07 & 168.33 \\
\hline SKO105 & 185.73 & 93.93 & 18.00 & 23.67 & 2.03 & 5.17 & 47.73 & 1.78 & 5.47 & 5.27 & 124.53 & 154.67 \\
\hline NDO25 & 119.47 & 101.07 & 11.87 & 32.33 & 2.40 & 6.21 & 44.60 & 1.78 & 5.47 & 5.40 & 129.13 & 167.73 \\
\hline JHO2007-2 & 119.80 & 94.07 & 11.60 & 31.00 & 2.10 & 6.75 & 47.27 & 1.79 & 5.20 & 5.60 & 125.80 & 162.73 \\
\hline CSAOSC14-6 & 165.27 & 93.80 & 15.93 & 36.00 & 2.90 & 7.12 & 47.40 & 1.59 & 5.40 & 5.20 & 123.80 & 156.67 \\
\hline
\end{tabular}




\begin{tabular}{|c|c|c|c|c|c|c|c|c|c|c|c|c|c|}
\hline SK0105 & \multicolumn{2}{|c|}{201.80} & 95.20 & 20.13 & 30.00 & 2.20 & 6.39 & 46.67 & 1.77 & 5.33 & 5.33 & 128.87 & 167.73 \\
\hline JHO2007-2 & \multicolumn{2}{|c|}{182.67} & 94.00 & 17.87 & 24.33 & 2.10 & 5.85 & 46.67 & 1.60 & 5.27 & 5.33 & 128.00 & 158.73 \\
\hline JHO03-93 & \multicolumn{2}{|c|}{143.67} & 84.93 & 13.67 & 34.33 & 2.57 & 7.67 & 44.67 & 1.63 & 5.33 & 5.20 & 124.93 & 169.13 \\
\hline ND0612 & \multicolumn{2}{|c|}{141.40} & 94.73 & 13.87 & 26.67 & 2.10 & 5.60 & 46.73 & 1.59 & 5.27 & 5.33 & 126.47 & 157.73 \\
\hline OS6 & \multicolumn{2}{|c|}{172.93} & 84.07 & 16.80 & 35.67 & 2.40 & 8.52 & 45.27 & 1.67 & 5.33 & 5.33 & 124.67 & 165.80 \\
\hline JHO851 & \multicolumn{2}{|c|}{141.20} & 94.73 & 13.80 & 31.67 & 1.70 & 6.96 & 43.80 & 1.63 & 5.27 & 5.33 & 125.27 & 159.33 \\
\hline JHO99-2 & \multicolumn{2}{|c|}{167.93} & 94.27 & 15.67 & 30.33 & 2.10 & 5.52 & 43.83 & 1.65 & 5.20 & 5.27 & 125.60 & 171.47 \\
\hline \multirow{2}{*}{ Range } & Min & 82.60 & 83.93 & 8.53 & 16.33 & 1.70 & 2.27 & 35.87 & 1.53 & 5.20 & 5.20 & 123.67 & 154.67 \\
\hline & Max & 221.60 & 100.13 & 20.13 & 35.67 & 2.90 & 8.52 & 50.07 & 1.81 & 5.47 & 5.53 & 131.07 & 177.93 \\
\hline Range of Variation & \multicolumn{2}{|c|}{$139 *$} & 16.2 & 11.6 & 19.32 & 1.2 & 6.25 & 14.20 & 0.28 & 0.27 & 0.33 & 7.4 & 23.26 \\
\hline
\end{tabular}

Contd.

\begin{tabular}{|c|c|c|c|c|c|c|c|c|c|c|}
\hline Variety & PL & BYPP & SSP & HSW & DWPP & HI & SG & SLL & SLDW & SVI \\
\hline CSOFSC12-2 & 26.37 & 14.13 & 93.60 & 4.83 & 10.81 & 26.96 & 65.40 & 25.27 & 0.03 & 2.30 \\
\hline CSOFSC11-5 & 26.50 & 13.93 & 95.07 & 3.43 & 10.68 & 26.93 & 83.27 & 19.23 & 0.02 & 1.82 \\
\hline Kent & 29.05 & 12.53 & 96.27 & 4.24 & 9.28 & 29.00 & 85.43 & 22.73 & 0.03 & 2.54 \\
\hline CSOFSC11-4 & 27.44 & 14.47 & 97.07 & 4.29 & 10.98 & 27.22 & 83.07 & 21.37 & 0.02 & 2.08 \\
\hline CSOFSC11-1 & 25.80 & 14.33 & 100.53 & 3.90 & 11.07 & 27.49 & 84.07 & 18.43 & 0.03 & 3.11 \\
\hline CSOFSC12-1 & 27.87 & 14.73 & 97.87 & 4.64 & 11.37 & 25.78 & 83.53 & 21.80 & 0.04 & 3.46 \\
\hline UPO212 & 27.53 & 16.33 & 106.20 & 3.72 & 11.10 & 25.97 & 84.20 & 28.47 & 0.02 & 1.78 \\
\hline ANDO1 & 24.93 & 14.27 & 102.27 & 4.45 & 10.23 & 29.61 & 86.67 & 18.60 & 0.03 & 2.10 \\
\hline JHO03-91 & 26.07 & 15.00 & 98.00 & 5.05 & 10.49 & 27.94 & 87.77 & 29.57 & 0.04 & 4.02 \\
\hline CSAOSC12-1 & 27.60 & 16.13 & 100.40 & 3.89 & 11.72 & 27.58 & 77.67 & 21.13 & 0.02 & 1.82 \\
\hline ANDO2 & 28.00 & 14.53 & 98.73 & 3.83 & 10.32 & 29.27 & 86.37 & 21.93 & 0.02 & 1.95 \\
\hline OS403 & 27.07 & 14.07 & 99.00 & 3.32 & 9.87 & 30.43 & 83.47 & 26.50 & 0.02 & 1.49 \\
\hline OS344 & 26.60 & 13.73 & 92.67 & 3.93 & 9.95 & 28.59 & 87.20 & 19.60 & 0.02 & 2.02 \\
\hline
\end{tabular}




\begin{tabular}{|c|c|c|c|c|c|c|c|c|c|c|c|}
\hline \multicolumn{2}{|c|}{ OS1 } & 23.60 & 14.73 & 88.73 & 3.21 & 10.12 & 28.69 & 84.27 & 19.40 & 0.02 & 1.89 \\
\hline \multicolumn{2}{|c|}{ SKO105 } & 27.33 & 13.27 & 92.35 & 3.48 & 9.23 & 31.92 & 87.07 & 21.97 & 0.03 & 2.64 \\
\hline \multicolumn{2}{|c|}{ NDO25 } & 25.93 & 13.53 & 90.60 & 3.95 & 9.12 & 34.09 & 81.17 & 22.53 & 0.03 & 2.46 \\
\hline \multicolumn{2}{|c|}{ JHO2007-2 } & 25.33 & 16.07 & 90.07 & 3.98 & 11.44 & 27.53 & 85.40 & 23.47 & 0.02 & 1.78 \\
\hline \multicolumn{2}{|c|}{ CSAOSC14-6 } & 26.00 & 12.67 & 91.93 & 4.84 & 8.68 & 30.75 & 88.23 & 24.47 & 0.03 & 2.48 \\
\hline \multicolumn{2}{|c|}{ SKO105 } & 27.13 & 13.00 & 95.00 & 4.63 & 8.94 & 33.84 & 90.77 & 29.97 & 0.03 & 2.76 \\
\hline \multicolumn{2}{|c|}{ JHO2007-2 } & 28.33 & 14.13 & 104.73 & 5.48 & 8.84 & 37.72 & 89.17 & 22.27 & 0.04 & 3.64 \\
\hline \multicolumn{2}{|c|}{ JHO03-93 } & 27.60 & 14.33 & 98.13 & 4.28 & 10.56 & 25.75 & 83.57 & 18.60 & 0.02 & 2.01 \\
\hline \multicolumn{2}{|c|}{ NDO612 } & 26.27 & 14.33 & 99.67 & 5.19 & 8.95 & 36.66 & 86.67 & 26.73 & 0.02 & 2.05 \\
\hline \multicolumn{2}{|c|}{ OS6 } & 19.33 & 13.53 & 88.27 & 4.97 & 9.38 & 33.90 & 84.20 & 21.03 & 0.03 & 2.54 \\
\hline \multicolumn{2}{|c|}{ JHO851 } & 17.13 & 13.67 & 92.40 & 4.99 & 9.47 & 30.91 & 87.57 & 23.20 & 0.03 & 2.83 \\
\hline \multicolumn{2}{|c|}{ JHO99-2 } & 17.27 & 14.07 & 91.07 & 3.73 & 9.78 & 27.88 & 85.53 & 28.00 & 0.02 & 1.59 \\
\hline \multirow{2}{*}{$\begin{array}{l}\text { Range } \\
\text { Range }\end{array}$} & Min & 17.13 & 12.53 & 88.27 & 3.21 & 8.68 & 25.75 & 65.40 & 18.43 & 0.02 & 1.59 \\
\hline & Max & 29.05 & 16.33 & 106.20 & 5.19 & 11.72 & 37.72 & 90.77 & 29.97 & 0.04 & 4.02 \\
\hline \multicolumn{2}{|c|}{$\begin{array}{l}\text { Range of } \\
\text { Variation }\end{array}$} & 11.92 & 3.80 & 16.46 & 1.98 & 3.04 & 10.91 & 25.37 & 11.54 & 0.02 & 2.43 \\
\hline
\end{tabular}


Analysis of GCV, PCV, ECV, heritability and genetic advance as percent of mean was observed for green fresh weight of total tiller GCV (21.43), PCV (23.52), ECV (217.51), heritability (82.95) and genetic advance as percent increase over mean (61.00). Days to $50 \%$ flowering was showed the values of GCV (4.91), PCV (4.94), ECV (0.25), heritability (98.84) and genetic advance as percent increase over mean (9.40).Total numbers of tillers per plant was exhibited value of GCV (20.15), PCV (21.61), $\operatorname{ECV}(1.27)$, heritability (86.90) and genetic advance as percent increase over mean (5.6). The GCV (16.52), PCV (20.87), ECV (12.81), heritability (62.70) and genetic advance as percent increase over mean (7.6) were observed for green plant weight. Green leaves weight per plant had the value of $\operatorname{GCV}(21.36)$, PCV(22.24), ECV (0.99), heritability (62.24) and genetic advance as percent increase over mean (2.1).The character stem girth, having the values of GCV(11.33), PCV (13.32), ECV (0.02), heritability(73.35) and genetic advance as percent increase over mean (0.40). The estimations of GCV (7.97), PCV (8.58), $\operatorname{ECV}(1.98)$, heritability (86.36) and genetic advance as percent increase over mean (6.8) were exhibited by leaf length of plant. The variability parameters viz; GCV (5.29), PCV (5.8), ECV (0.01), heritability (83.27) and genetic advance as percent increase over mean (0.2) were recorded from the Leaf width of plant. Nodes per plant were displayed an estimates for GCV (0.01), PCV (3.18), ECV (0.03), heritability (17.41) and genetic advancement percent increase over mean (0.1). Leaves per plant having the values of GCV (0.01), PCV (3.59), ECV (0.04), heritability (6.65) and genetic advancement percent increase over mean (0.01). Days to maturity had estimates for GCV (1.84), PCV (1.86), ECV (0.09), heritability (98.33) and genetic advance as percent increase over mean (4.8). The GCV (4.05), PCV (4.42), ECV (8.89), heritability (83.82) and genetic advance as percent increase over mean (12.80) were estimates for plant height.

Panicle length was showed the values for GCV (12.23), PCV (12.55), ECV (0.51), heritability (95.07) and genetic advance as percent increase over mean (6.3). Biological yield per plant was displayed an estimates of GCV (6.71), PCV (6.84), ECV (0.04), heritability (96.15) and genetic advance as percent increase over mean (1.9). For seeds per plant GCV (5.01), PCV (5.14), ECV (1.12), heritability (95.38) and genetic advance as percent increase over mean (9.7) variability components were estimated. The 100-seeds weight was exhibited the estimates of GCV (14.34), PCV (15.86), ECV (0.08), heritability (8.75) and genetic advance as percent increase over mean (1.10). Dry weight per plant was showed with values of GCV (8.89), PCV (9.20), ECV (0.06), heritability (93.38) and genetic advance as percent increase over mean (1.8). An estimation of GCV (11.09), PCV (11.37), ECV (0.53), heritability (95.30) and genetic advance as percent increase over mean (6.6) was observed for harvest index in percent.The seed germination in percent was expressed with values of GCV (7.27), PCV(8.76), ECV(47.45), heritability (13.29) and genetic advance as percent increase over mean (2.0). The estimates of GCV (14.84), PCV (15.77), ECV (1.5), heritability (88.62) and genetic advance as percent increase over mean (6.6) were recorded for seedling length. The single seedling dry weight was expressed the values for GCV (24.29) $\mathrm{PCV}(27.79), \operatorname{ECV}(0.00)$, heritability (76.37) and genetic advance as percent increase over mean (0.0). Seed vigour index had the values of GCV (26.38), PCV (29.81), ECV (0.11), heritability (78.3) and genetic advance as percent increase over mean (1.1) in present investigation. The seed yield per plant was displayed an estimations for GCV (9.72), PCV (9.96), ECV (0.01), heritability (95.28) and genetic advance as 
percent increase over mean (0.8) in this study. A crossing between genotypes having higher values for the useful traits will helps an improvement of characters and in development of new genotypes with increase in grain and fodder yield. (Khan et al., (2002), Pundir et al., (2008), Hossein et al., (2011), Bibi et al., (2012), Krishana et al., (2013), Wani et al., (2013) Dubey et al., (2014), Bajpai et al., (2014), Kumar et al., (2017) and Singh et al., (2018). A close resemblance between the corresponding estimates of phenotypic coefficient Variance and genotypic coefficient variance suggested little role of environment in the expression of different traits.

It is evident from the table 3 that phenotypic variances ranging between leaf width (0.001) and green fresh weight (1275.96) and genotypic variances ranging between leaf width (0.001) and green fresh weight (1058.44) for these traits whereas, phenotypic coefficients of variation (PCV) ranged from 1.86 for days to maturity to 29.81 for seed vigour index, considered in this study. Deshmukh et al.,(1986) classified PCV and GCV values as high (>20\%), medium (10 $20 \%)$ and low (<10\%). Seed vigour Index (29.81) showed high value of PCV followed by single seedling dry weight (27.79), green fresh weight of total tillers (23.52), green leaves weight per plant (22.24), total tillers per plant (21.61) and green plant weight (20.87),comparatively higher phenotypic variance value of 1275.96 for green fresh weight of total tillers was recorded in this study, similarly, the genotypic variances for this character almost as high, indicating that the genotype could be reflected by the phenotype and the effectiveness of selection based on the phenotypic performance for these characters. Bhal et al., (1988), Dubey et al., (1995), Choubey and Gupta (1986) and Choubey et al., (1986). However, medium PCV and GCV were displayed in medium was displayed in 100- seed weight (15.86), single seedling length (15.77), stem girth (13.32), panicle length (12.55), harvest index (11.37). Bhal et al., (1988), Dubey et al., (1995), Choubey and Gupta (1986) and Choubey et al., (1986). Furthermore, low PCV and GCV, were observed for seed yield per plant (9.96), followed by dry weight per plant (9.20), seed germination in percent (8.76), leaf length (8.58), biological yield per plant(6.84), leaf width (5.80), seeds per plant (5.14), days to flowering (4.94), plant height (4.42), leaves per plant (3.59), nodes per plant (3.18) and days to maturity (1.86), indicating minute scope of selection as they are under the influence of environment. Wide differences between PCV and GCV values were observed in nodes per plant, leaves per plant, which may be indicate significant influence of environmental factors on these characters, thus, it would be appropriate to consider the above characters depending on the objective of crop improvement program.

Pramoda and Prasad (2007) divided heritability estimates as low $(<40 \%)$, medium (40-59\%), moderately high (60-78\%) and very high $(>80 \%)$ whereas, genetic advance was grouped by Johansson et.al.,(1955a) as $>20 \%$ high, $10-20 \%$ moderate and $<10 \%$ low. Heritability estimates varied from $6.65 \%$ for leaves per plant to $98.84 \%$ for days to maturity.

High to moderately high variability for PCV and GCV coupled with high estimates of heritability were recorded for total numbers of tillers per plant (86.90) followed by green fresh weight of total tillers (82.95), green plant weight (62.70), green leaves weight per plant (62.24), stem girth (73.35), panicle length (95.07), harvest index (95.30) and single seedling length (88.62) present in Table 3, which indicate an advantage through simple selection. Khan et al., (2002), Pundir et al., (2008), Hossein et al., (2011), Krishana et al., (2013), Wani et al., (2013) Dubey et al., 
(2014), Bajpai et al., (2014), Kumar et al., (2017), Kumar et al., (2017) Sarojini et al., (2017) and Singh et al., (2018).

\section{References}

Allard, R.W. (1960). Principles of plant breeding. John Willey and Sons Inc. New York, 485p.

analysis of quantitative traits in Virginia bunch varieties of groundnut Indian J. Agri.Sci., 56: 515-518.

Annonymous (2016) Published report on oat by IGFRI Jhansi

B. A. Wani, M. Ram, Abrar Yasin. B, Majid Ali, Ashiq Pandith and Raouf A. Mir (2013)Seedling vigour in wheat (Triticum aestivum L.) as a source of genetic variation and study of its correlation with yield and yield components African Journal of Agricultural Research Vol. 8(4), pp. 370-372,

Bajpai, Ruchi Singh, Priyanka and Singh, Poonam (2014) Study on Seed Vigour and Their Correlation to Field Emergence in Groundnut (Arachis Hypogea L.) Genotypes Agricultural Science. Volume: 35, Issue: 144-150.

Bibi, shahzad, A.N., Sadaqat, H., Tahir M.H.N. and Fatima, B.(2012). Genetic Characterization and inheritance studies of oats (Avena sativa L.) for green fodder yield. Int.J.Bio. pharmacy and Food. Sci., 1(4): 450-460.

Burton, G.W. (1952). Quantitative inheritance in grasses. Proc. $6^{\text {th }}$ Int. Grassland Cong.1:227-283

Deshmukh, S,N.N., M.S. Basu and Reddy, (1986). Genetic variability, character association and path coefficient

Dubey, Nidhi Avinashe, H.A. Samidha Jaiwar and Chichkhede, L. (2014) Characterization and inheritance studies of oats (Avena Sativa L.) For green fodder yield. Int.J.Bio.Pharmacy and Food. Sci., 1 (4): 450 (Accepted: 02 Aug 2014).

Hasan, C.H., H. F. Robinson and R. E. Comstock (1956). Biometrical studies of yield in segregating populations of Korean espediza. Agron. J. 48: 268272.

Hossein Aliabadi Farahani, Payam Moaveni and Kasra Maroufi (2011) Effect of Seed Size on Seedling Production in Wheat (Triticum aestivum L.) Advances in Environmental Biology, 5(7): 1711-1715.

Johnson HW, HF Robinson and RE Comstock (1955a). Estimates of genetic and environmental variability in soybeans, Agron. J. 47: 514-318.

Khan MQ, Anwar S, Khan MI (2002). Genetic variability for seedling traits in wheat (Triticum aestivum L.) under moisture stress conditions. Asian J. Plant Sci. 5:558-90.

Krishna, Abhishek, Shahid Ahmed, Pandey H.C. and Bahukhandi, D. (2013) Estimates of genetic variability heritability and genetic advance of oat genotypes for green fodder yield Agricultural Science Research Journals Vol. 3(2), pp. 56-61.

Kumar, Ajay, Prakash, J. Gupta and Kumar, Pankaj (2017) Performance of wheat varieties regarding seed quality parameters Journal of Pharmacognosy and Phytochemistry 2017; 6(4): 12211223.

Kumar, Prem Nirmala Kumara R.,A. and Ananda Kumar, C.R. (2017). Studies on Genetic Variability and Character Association among Yield and Yield Attributing Traits in Oats (Avena sativa L.). Int.J.Curr.Microbiol. App.Sci. 6(11): 4075-4083.

Mahatma Sarojini, Ratan Narayan and Viswanathan Naidu (2017)An evaluation on genetic variability in seedling vigour in wheat (Triticum 
aestivum L.) Advances in Seed Science and Technology Vol. 1 (3), pp. 018020.

Peterson, D.M., Wesenberg, D.M., Burrup, D.E. and Erickson, C.A. (2005). Relationships among agronomic traits and grain composition in oat genotypes grown in different environments. Crop Sci., 45: 1249-1255.

Pramoda, H.P. and Gangaprasad, S. (2007). Biometrical basis of heading segregation population for Improving productivity in onion (Allium cepa $\mathrm{L}$.) J. Asian Horti., 3: 278-280.
Pundhir S.R.,V.P. Singh, and Phogat, D.S. (2008). Genetic variability and interrelationship for various grain and seedling characters in oats, Forage Res. 33: 236-240.

Singh, Atar Vyas, R.P. Singh, H.C. Kumar, Sarvendra Deep, Amar Malik, Piyush and Singh, Akash (2018) Genetic variability and correlation of seed yield and related characters in oat (Avena sativa L.) International Journal of Chemical Studies 2018; 6(1): 15331537.

\section{How to cite this article:}

Atar Singh, Mayank Chaudhary, Nirdesh K. Chaudhary and Chiranjeev. 2019. Genetic Analysis for Forage Yield and Morphological Traits of Seed in Oat (Avena sativa L.). Int.J.Curr.Microbiol.App.Sci. 8(10): 128-142. doi: https://doi.org/10.20546/ijcmas.2019.810.014 